\title{
Detection of Bacterial Canker Disease In Mango Using Image Processing
}

\author{
Amritha S Nadarajan ${ }^{1}$, Prof.Thamizharasi $\mathrm{A}^{2}$ \\ ${ }^{1}$ M.Tech Computer Science and Engineering, Mohandas College of Engineering and Technology, Trivandrum, \\ Kerala, India \\ ${ }^{2}$ Assistant Professor, Department of Computer Science and Engineering, Mohandas College of Engineering and \\ Technology, Trivandrum, Kerala
}

\begin{abstract}
Mango is considered as the king of fruit. It is a leading fruit in our country. It is considered as the national fruit of India. It has good flavour and also provide vitamin C. Mangoes are considered as to be the king of the fruit. India has the richest collection of mango cultivars. This work detects the external bacterial disease in Alphonso mango using image processing technique. The detection of diseases in mango is very difficult by our human naked eyes. It needs more time and cost. The manual detection of bacterial fruit disease is not simple and not accurate. The paper proposes a digital image processing method for the detection of external bacterial disease in Alphonso mango. Alphonso variety of mango is chosen for this work. The sample input of mango is collected from DB_MANGO set which is publicly available in internet. The proposed system has three phases. First, the image is acquired using a digital camera with a specified image size. The input image is preprocessed using histogram stretching method. The colour features of mango fruit are extracted using RGB colour pattern. The second phase is to segment the affected areas from the normal fruit. The watershed algorithm is used for segmentation. The third phase is the feature extraction and classification of defects part of an image by using a template matching algorithm. The output of the proposed system identifies whether the mango fruit is normal or diseased fruit.
\end{abstract}

Keywords: Bacterial canker disease detection, segmentation, feature extraction, classification, mango, preprocessing.

\section{Introduction}

Mango is considered as the king of fruit. Mango is considered as the national fruit of India because it is a leading fruit in India. It has good flavour and also provide vitamin C. Good mangoes pulps are very important items of export from India. Kuwait, UAE and Middle East countries are the main mango export destinations. There is a good scope for improving the marketing field and also production of mango in our country. It has great economic importance in the marketing field.

The quality of a fruit is based on several factors such as sweetness, nutritional value, colour and size. A fleshy mango fruit contains more than $80 \%$ of water. The size of the fruit always depends on the water content inside the fruit. Now, 30 different varieties of mango are grown, and Alphonso is the important one in rating.

Alphonso is the best variety of mango in the world. It fetches premium in marketing. Alphonso is the one of the best seasonal mango. It has good flavour, sweetness. It is named as "Alfonse de Albuquerque". Portuguese contributes a good variety of mango through the grafting of mango trees. It is an expensive variety of mango in the world. According to the colour change in fruit, we can easily identify the diseased fruit or not. Manually disease identification in mango is costly and also time consuming. The most important diseases affected in mangoes are like fungi, bacteria diseases etc.

Identification of fruit disease leads to prevent the disease in the earlier state and increasing the productivity of good quality mangoes. In this paper, we propose an approach for the detection of diseased mango fruit using digital image processing techniques [1].This approach helps to improve the marketing of fruit production. Small sized disease which is not identifiable through computerized detection. Detection of the disease in on time and treated in on time can reduce the fruit diseases and improving the quality of the fruit. Detection of bacterial disease through manually is not accurate. In the proposed work the computerized vision technique is used to help automatic detection of disease.

In this work disease detection is done through digital image processing method. The basic image processing steps like preprocessing, segmentation, classification are used in this work. In this work the input samples are collected from the internet (DB_MANGO set) and mainly experiments are done in an Alphonso mango variety. The proposed work consists of three phases; the first phase is the input image from the data set for preprocessing; the second phase is using a watershed algorithm for segmentation; the third phase is template matching algorithm is used for feature extraction and normalized classifier is used for classification. 


\section{Literature Survey}

Shivram dubey and Anand Singh Jalal proposed an approach for the detection of disease in fruit. Basic image processing techniques are used for the detection. They used K-means clustering for segmentation. The author considered the diseased apple. The work mainly concentrated on three types of apple diseases, namely apple scab, apple blotch and apple rot. The work shows the accurate detection of the fruit diseases [2].

Leila Farahani and Hasan Reza Etebarian proposed an approach for the identification of disease blue mouth on apple using the RGB components. The image processing and CVS (Computer Vision System) are used for ordering the fruits. It provides the efficiency and accuracy of quality of the fruit [3].

Garbriel Leivaa and German Mondragonbet, proposed a simple and cheaper method for implemented to clear unities with fungal damage. Segmentation techniques are used to segment anthracnose diseases on mango, and a neural network classifier is used to classify normal and diseased fruits Considered three types of fruit namely mango, grape and pomegranate. The work consists of two phases. In the first phase, thresholding is used for segmentation; region growing, K-means clustering and watershed are used for identifying anthracnose affected areas from normal fruit. Then these affected areas are graded by calculating the percentage of affected area. The second phase texture features are extracted using Run length Matrix. ANN classifier is used for classification. The work has to categorize blueberries with fungal decay, and damages [4].

Jagadeesh Dev Das Pujari and Rajesh Yakkundimath et al considered three variable types of fruit. Mango, grape and pomegranate are the data samples. The areas affected by anthrax are segmented and graded, based on percentage of the affected area and classify the normal and diseased fruit. Neural network classifier is used for classification [5]. Suvarna Kanakaraddi and Prashant Iliger proposed the method for identifying the chilly disease. The method is used for increasing the production and reducing the human error. The basic image processing technique is used in this work. Classification is based on the percentage of affected portion [6].

C.C.Teoh and A.R.Mohd Syaifudin proposed a study on weight measuring of chokanan mangoes. Image processing and analysis technique is used for the weight measurements. Regression was used to analyse the relationship between mango pixel and weight [7]. R. Yogamangalam and B. Karthikeyan has presented an outline of most common segmentation techniques. They describe about thresholding, model based, Edge detection clustering, etc. It describes merits and demerits of segmentation techniques and also discussed about the Markov Random Field [8].

\section{Existing System}

In the existing system, Pujitha N, Swathi C and Kanchana V proposed a method for detection of external defects on mango. The work implemented in mango fruit .They collect the input sample as in video format and again converted into 100 frames. Several methods are used for the detection of defects in mango. This work implemented by digital image processing technique. Digital image processing technique consists of preprocessing, segmentation, feature extraction, classification and recognition.

\subsection{Methodology}

There are various techniques for detecting the disease on mango that involves colour or shape based. We use digital image processing techniques to find the diseased area.

The steps are as follows

1) Input the video of the mango.

2) Preprocessing.

3) Image segmentation using Watershed algorithm.

4) Feature extraction using Template matching algorithm.

The mango image is taken as the input for the preprocessing phase. It enhances the image clarity and reduces background noise. Thresholding method is used for the preprocessing of images. Preprocessing phase, convert the original image into binary image and perform the individual RGB colour bands by computing histograms. Finally, displaying the colour threshold ranges through histograms.

Segmentation technique is the process of dividing the image of mango into different segments. Segmentation is applied to detect the affected area. The segmentation of images is done by using the watershed transformation algorithm. It is used for automatic detection of images used to segment the affected areas from normal area of the mango. Segmentation is classified as follows: Region based, Edge based, Threshold, feature based, clustering, and Model based. Watershed segmentation technique is to find in the region of highest gradient intensity. The feature extraction of defected part of the image is done by based on region detection method. The required features are extracted from the images. It is mainly used for finding the defects portion of the mango. The feature extraction is done by using a template matching algorithm. It uses normalized crosscorrelation for the segmented area. The accuracy and efficiency of the system are validated for finding the pixel intensity value. 
The Different back spot on the image is categorized based on pixel intensity by using the blob extraction method. The classification is done by matching the feature of object and template feature. Template matching method is used for both feature extraction and classification. The classification is performed by correlation that takes less time to recognize objects in an image and display the defected region of the fruit. Grading is performed which helps to understand the quality of the fruit. They proposed the future work includes working with large dataset and detecting using the same methods with other variety fruits.

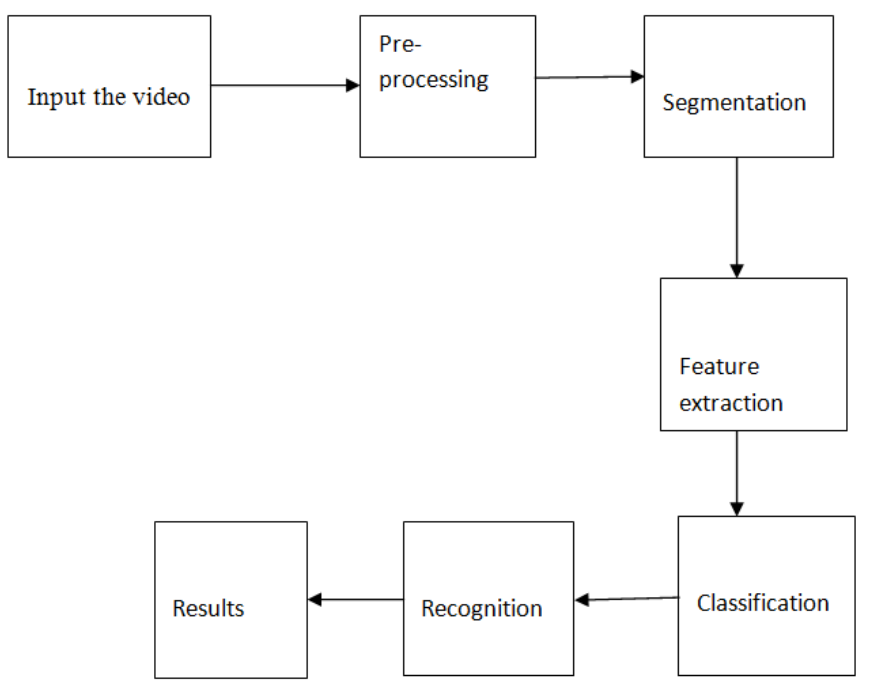

Fig.1 Architecture of the existing system

Segmentation is used for identifying the defected region of the fruit and give remedies to prevent the fruit from the diseases. Classification is performed by correlation. It needs less time to detecting the diseased region of the fruit. Grading methodology is very helpful to farmers for identifying the diseased fruit and take preventions. Fig. 1 shows the architecture of the existing system.

\subsection{Introduction}

\section{Proposed System}

Mangoes are said to be the king of all fruits in the entire world. Mango is the delicious seasonal fruits grown in the tropical areas. Depending upon cultivar, mango comes in different shapes and sizes. Its flavour is rich and good and sweet with a mild tartness. Mango fruit is a source of Vitamin-A. The defects in the fruits can be identified easily with the difference in the colour that appears when compared to the normal fruit.

\subsection{Motivation of the study}

The work is implemented in Alphonso mango. It is a seasonal fruit. It is also one of the most expensive varieties of mango.The work mainly concentrated on the bacterial disease on Alphonso mango. $X A N T H A M O N A S$ CAMPESTRIS /Bacterial canker is the one of the main bacterial disease affected on Alphonso mango. The objective of the work to deduct the "XANTHAMONAS CAMPESTRIS" disease in mango (Alphonso). The proposed work consists of normal image processing concept image preprocessing, segmentation, feature extraction and classification. In this work the input images are in JPEG format. Input sample are taken from the DB_MANGO dataset from the internet. The experimental work is done in Alphonso variety. The bacterial canker disease is rapidly spreading during rainy days. The disease is spread through the infected planting parts. When the fruits are in bunches the bacterial disease is spread through the contact with each other. The disease is found on leaves, stem, branches and fruits. This disease is mainly seen in the form of irregular necrotic patches (cankerous patches) with brown colour. On fruits, they appeared in water soaked, brown colour lesions.

Alphonso is in great demand on abroad. The export of fresh mangoes has been showing an increasing trend, with earnings from export of fresh mangoes in (2014-2015)-30253.65 lakhs, Major export destinations for our mangoes are Gulf countries (51\%), Bangladesh (33\%), European Union (10\%). Among the Indian varieties, Alphonso is more demand in abroad. Special features of Alphonso mango are the leading commercial variety of Kerala and one of the excellent varieties of the country. The fruit is medium in size, oblique in shape and yellow in colour. Slightly acidic, good flavour, long life to withstand transport and early bearing; and also have to enhance its competitiveness over other varieties. Alphonso has excellent taste; size and colour do not match varieties of other countries. 


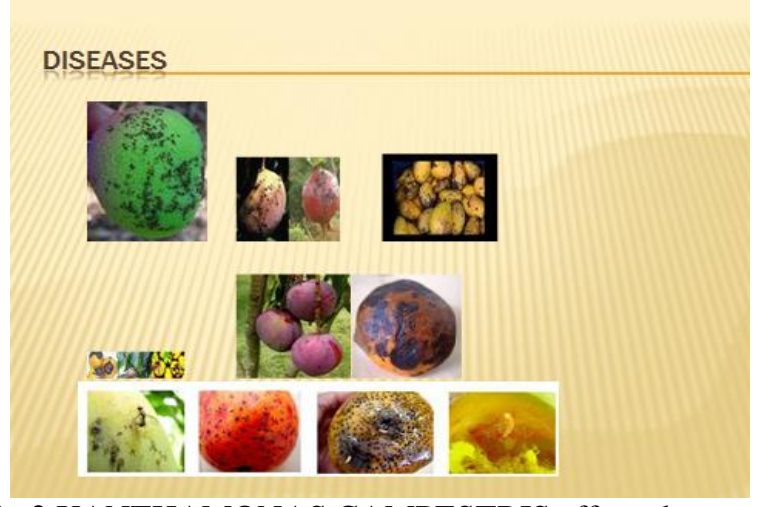

Fig.2 XANTHAMONAS CAMPESTRIS affected mango.

The block diagram of the proposed system is shown in fig. 3

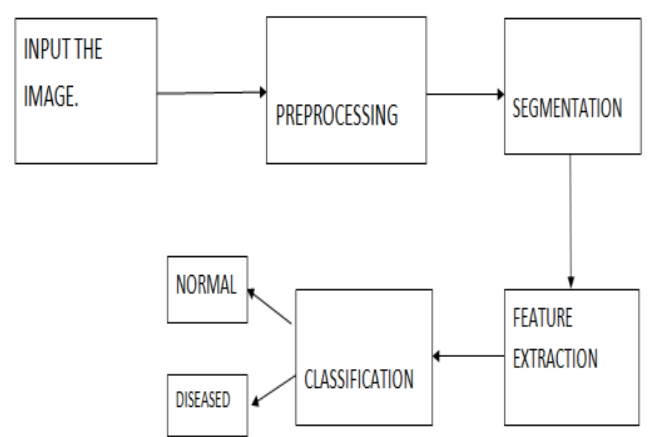

Fig.3 Architecture of the proposed system

In the proposed system, preprocessing is done for remove background noise and increasing image quality. The identification of diseased portion is done by applying segmentation.

\subsection{Algorithm}

The proposed work uses three algorithms:

$>$ Algorithm for image extraction and preprocessing.

$>$ Algorithm for segmentation.

$>$ Algorithm for feature extraction and classification.

In preprocessing phase, each image is taken to detect the defected fruit, so the quality of the image is improved and reduces the noise and improve the feature of the image [15]. In this phase to convert the input image into HSV image.

\section{Algorithm}

Step 1: Insert the mango images.

Step 2: Read the image into the opencv in c sharp language from a specific location.

Step 3: Convert the input image into hue, saturation and value image (HSV).

Step 4: Select the colour ranges for thresholding and display through histograms.

Step 5: The filtered mask is applied to the original image (RGB image).

Step 6: Finally, Displaying the masked image in specific colours.

In segmentation phase, dividing them into different segments. Before segmentation apply thresholding for getting the binary image with black and white format. Segmentation are different types, i.e., Edge based, Region based, Threshold based etc. In this work watershed segmentation technique is used. In feature extraction and Classification phase, region detection is used for feature extraction which is used for finding the defect portion of the image .In this work feature extraction is performed by using a template matching algorithm.Normalized cross-correlation is used for identification of segmented area for detecting the template with images. The different small spots are separated based on the intensity of the pixel, with the help of the blob extraction method.The normalized Co relation method is used to correlate both feature extraction and classification. 


\section{Experimental Results}

The input images are collected from DB_MANGO dataset available in the internet is shown in fig.5.
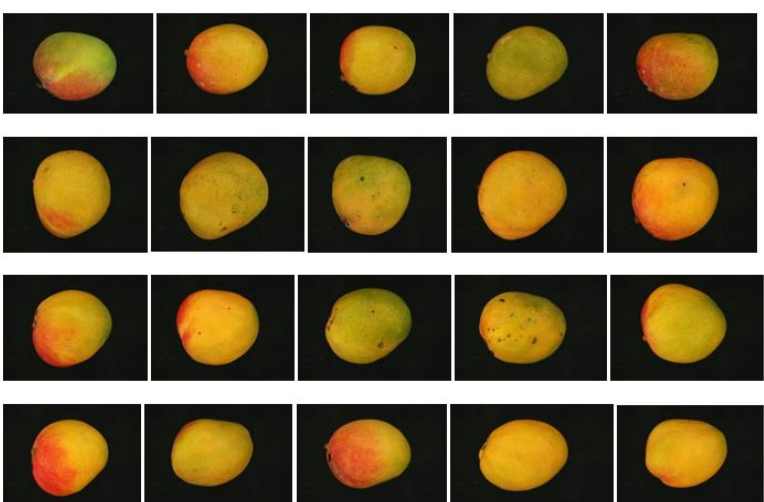

Fig.5 The images of mango in DB_MANGO set.

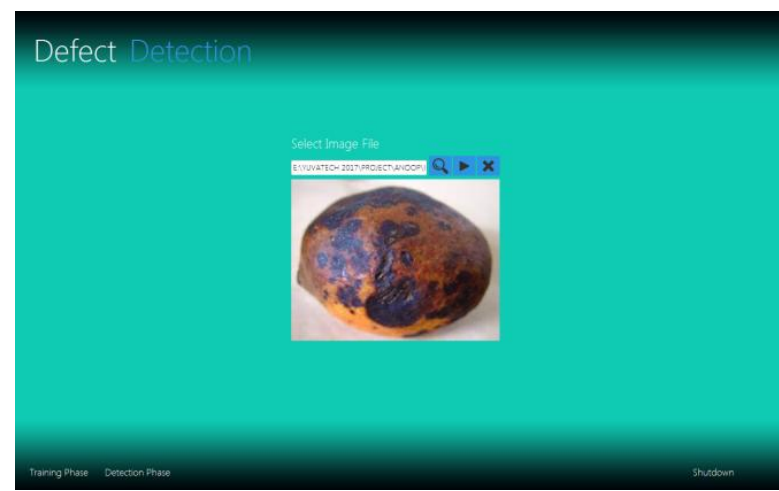

Fig.6 Screenshot of image selection.

Fig.7 Histogram of the color features of the input image.'
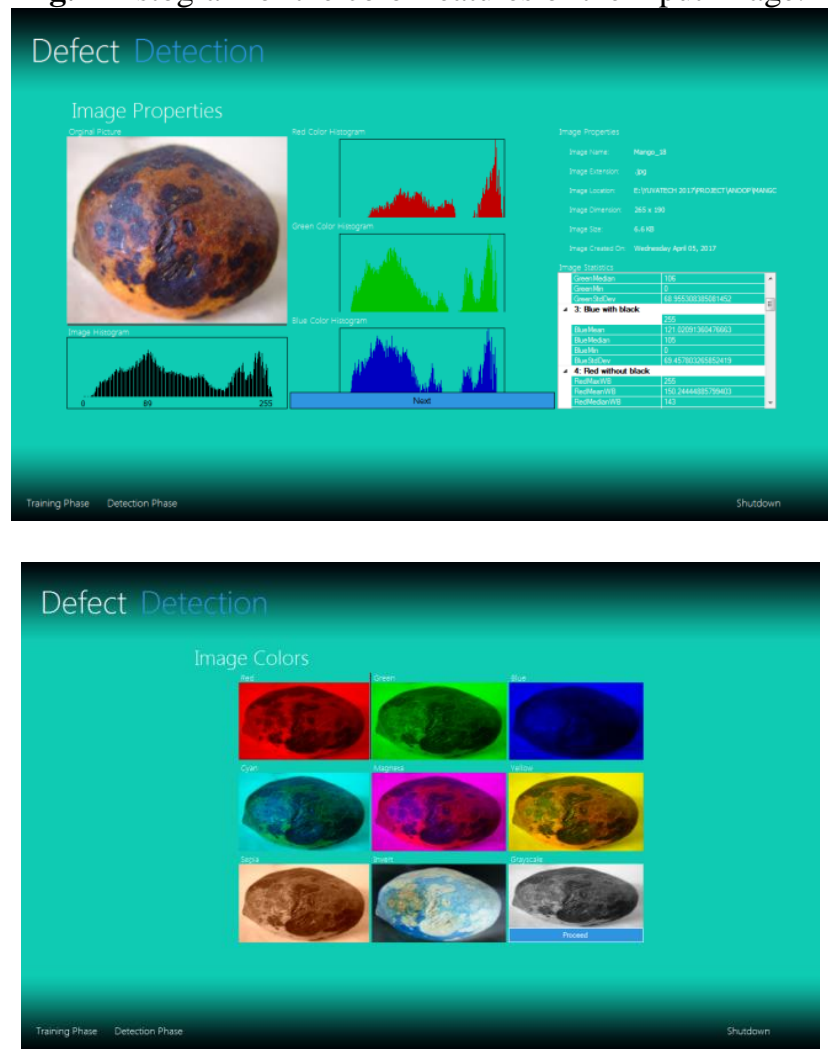

Fig.8. Image representation after thresholding. 


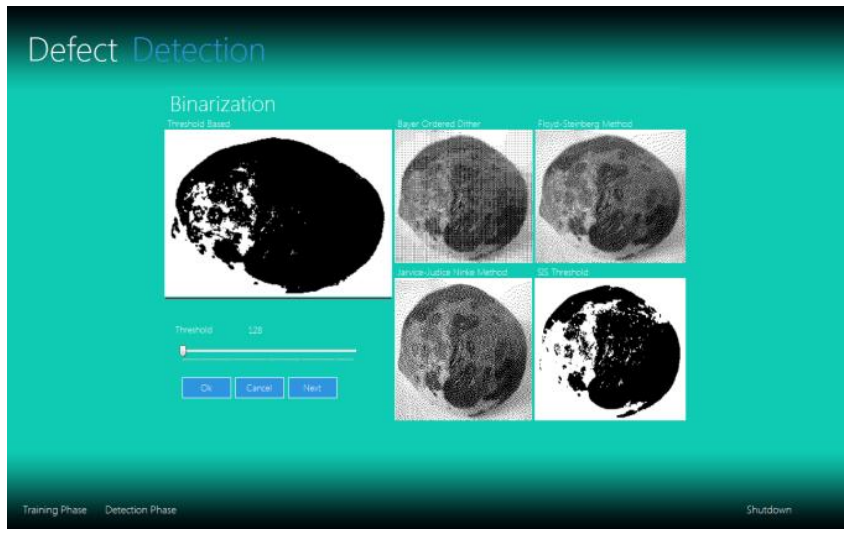

Fig.9.Binarization of the input image.

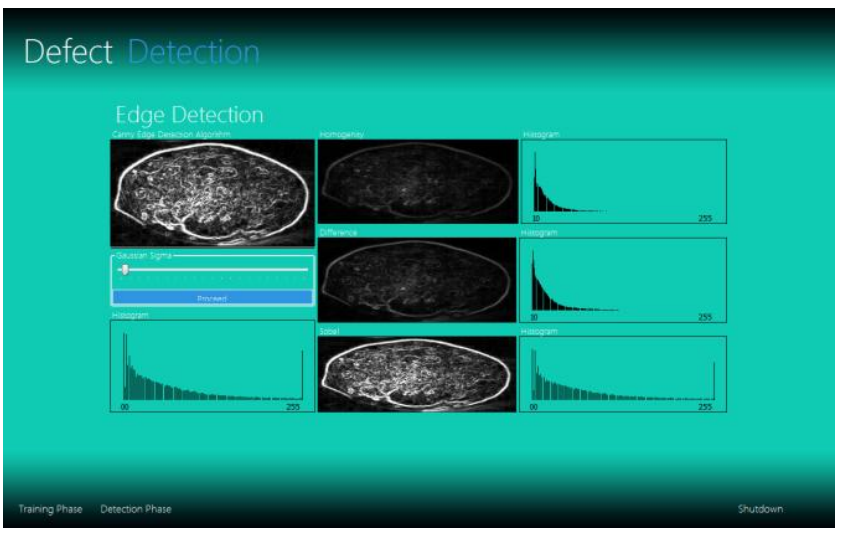

Fig.10.Edge detection of input image.

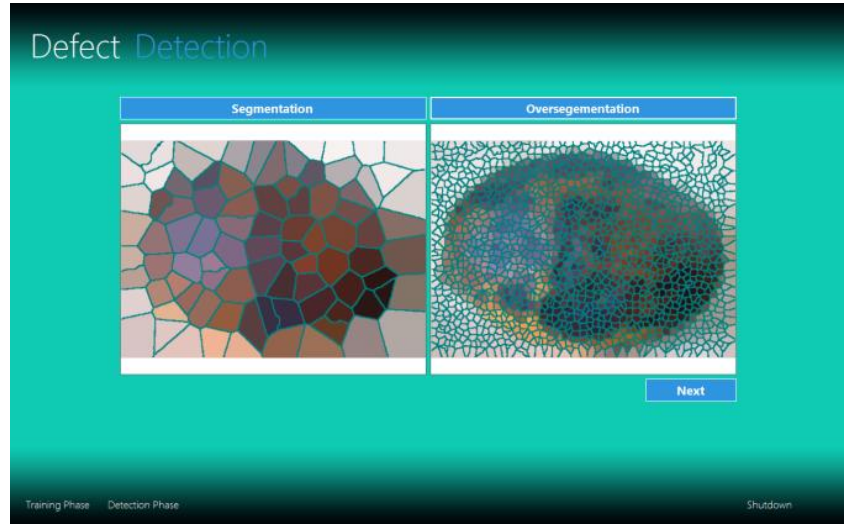

Fig.11.Segmentation using watershed algorithm.

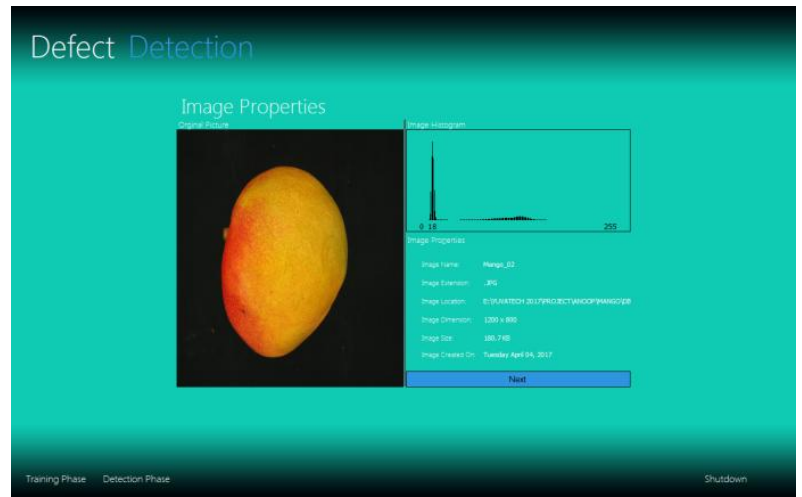

Fig.12.Detection phase. 


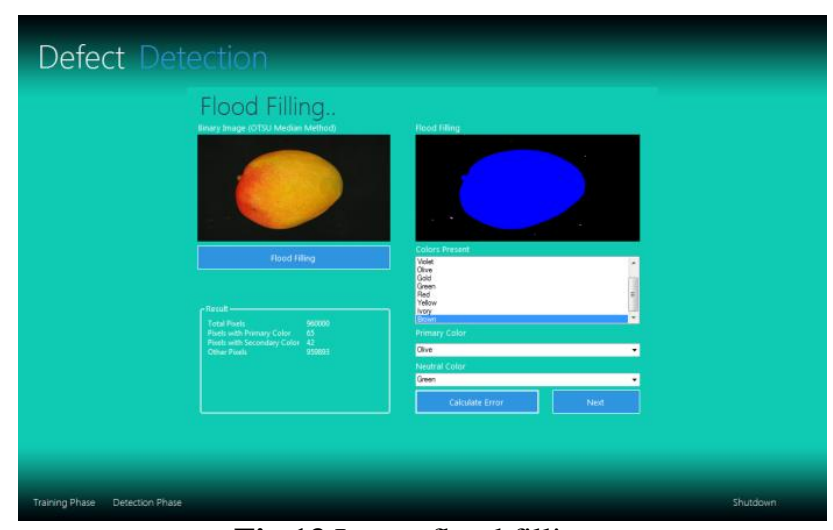

Fig.13.Image flood filling.

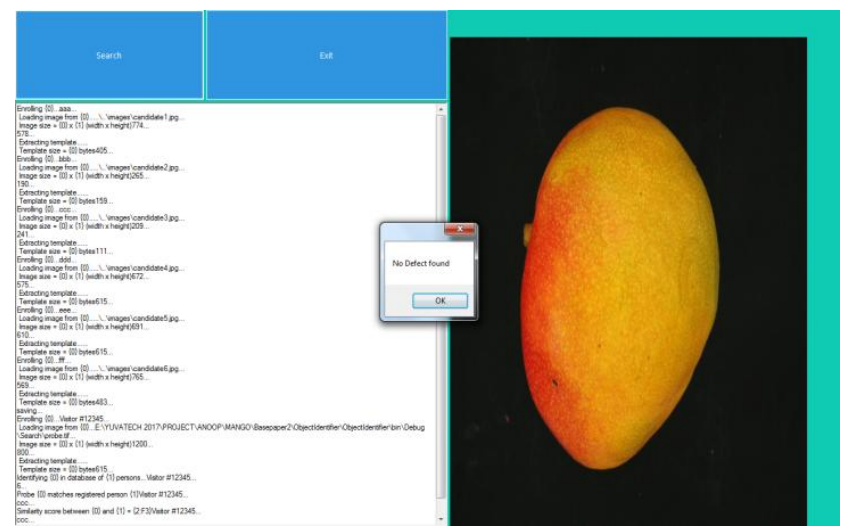

Fig.13.Detect as normal fruit.

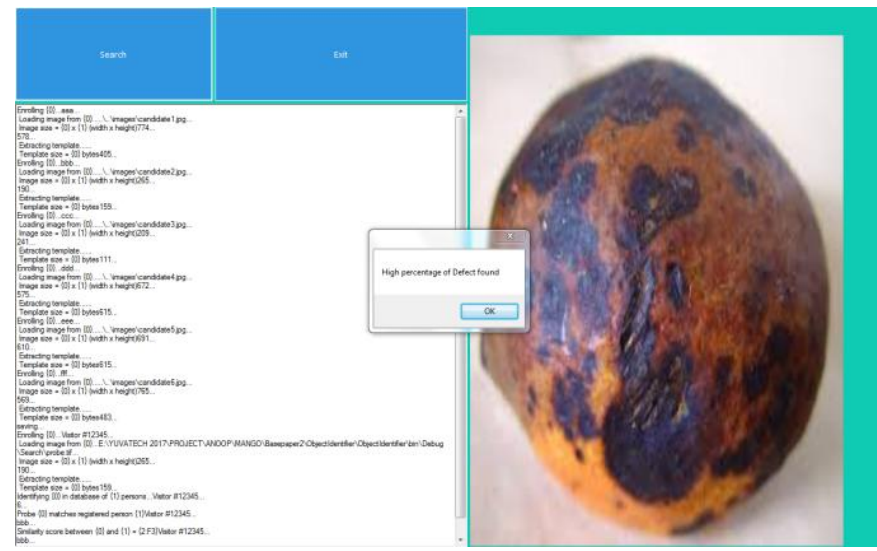

Fig.14.Display the input mango fruit is diseased.

\section{Conclusion}

In this paper the disease identification in Alphonso mango is described by applying the digital image processing technique. In this paper three methods are discussed. The implemented technique is beginning of image acquisition, preprocessing and histogram representation of the input image. The proposed work focuses on external bacterial disease Identification on Alphonso mango. Based on the colour of the image the intensity of the defect area is predicted. The work simply identifies whether the given mango is normal or diseased.

\section{References}

[1]. Pushpa B R, MeghanaT K, TripullaK H(2015)“Detection and classification of fungal disease in fruits using imageprocessing technique"in International journalof applied engineering research(IJAER), Vol 10, number 55.

[2]. Shiv Ram Dubey, Anand Singh Jalal (2012) “Adapted Approach for Fruit disease Identification using Images", in International Journal of computer vision and image processing (IJCVIP) Vol 2, no. 3:44-58. 
[3]. Leila Farahani, Hasan Reza Etebarian, Hadis Mohseni Takallou, Navazola Sahebani, Heshmatolah Aminian, (2012) “Computerbased recognition of severity of apple blue mold using RGB components”, International Research Journal of Applied and Basic Sciences, ISSN 2251-838X, Vol., 3 (1).

[4]. Gabriel Leivaa, Germán Mondragónb, Domingo Meryb, José Miguel Aguileraa, (2011) "The automatic sorting using image processing improves postharvest blueberries storage quality", Department of Chemistry and Bioprocess Engineering, Pontifical Catholic University.

[5]. Jagadeesh Dev Das Pujari, Rajesh Yakkundimath and Abdulmunaf Syedhusain Byadgi March, 2013 "Grading and classification of anthracnose fungal disease of fruits based on statistic texture features"in International Journal of Advanced Science and Technology Vol. 52,.

[6]. Suvarna kanakaraddi, Prashant iliger, Akshay gaonkar, Minal alagoudar, Abhinav Prakash, computer science, engineering department, b. v. b. c. e. t Hubli, India, (20 Apr 2014), "analysis and grading of pathogenic disease of chili fruit using image processing", proceedings of international conference in advance in engineering and technology, India, ISBN: 978-93-84209-063.

[7]. C. C. Teoh and A. R. Mohd Syaifudin, (2007), "Image processing and analysis techniques for estimating weight of Chokanan mangoes", J. Troop. Agric. And fed. Sc. 35(1) 183-190.

[8]. R.Yogamangalam,B.Karthikeyan(2013), "Segmentation Techniques Comparison in image Processing", International Journal of Engineering and Technology (IJET), FEB, VOL 5 ISSUE 1, P307. 